\title{
ASMA EN NIÑOS: UNA REVISIÓN EN TORNO A LOS CAMBIOS EN SU TRATAMIENTO
}

\author{
ASTHMA IN CHILDREN: A REVIEW OF CHANGES IN TREATMENT
}

Dr. Luis Enrique Vega-Briceño ${ }^{1}$

1.- Pediatra Broncopulmonar, Clínica Alemana de Santiago. Profesor Asociado UDD. Asesor Médico GSK

\section{INTRODUCCION}

El asma leve-moderada se define según la frecuencia de síntomas y el riesgo de presentar una exacerbación según la Guía de la Iniciativa Global para el Asma (CINA) y el Informe del Panel de Expertos del Programa Nacional de Educación y Prevención del Asma de los Estados Unidos del Instituto Nacional del Corazón, Pulmón y Sangre (NHLBI) $(1,2)$. Otra definición considera el nivel de control de síntomas de asma mediante el uso regular de controladores (1). Si bien los pacientes con asma leve-moderada representan $>70 \%$ de la población con asma, son un grupo muy heterogéneo y complejo (3). Los pacientes con asma leve tienen riesgo de desarrollar exacerbaciones severas (0.12-0.77 exacerbaciones severas/paciente/año) definidas, tradicionalmente, por el uso de esteroides orales, visita a urgencia u hospitalización; paradójicamente representan el grupo con la peor adherencia al uso regular de controladores (4).

El riesgo de una exacerbación severa en pacientes con asma leve despertó la preocupación y promovió el desarrollo de numerosos estudios clínicos durante los últimos 15 años con el propósito de reducir estas cifras alarmantes e incluso el riesgo de muerte asociado; ello culminó con la introducción de numerosos cambios en las nuevas estrategias y recomendaciones. Durante el 2019 se introdujo una interesante recomendación en torno al uso de una combinación fija de un corticoide inhalado (ICS) más formoterol (FT) en un sólo inhalador -Budesonida (BUD)- como tratamiento de mantenimiento y rescate al mismo tiempo, denominado rescate anti-inflamatorio, con el propósito de superar los problemas de adherencia y disminuir el riesgo de exacerbaciones severas en adolescentes y adultos.

Por otra parte, a principios del año 2020 se incluyó una alerta y recomendación de la Administración de Alimentos y Medicamentos (FDA) sobre el riesgo de efectos adversos neuropsiquiátricos graves relacionados con el uso de montelukast como controlador en niños y adolescentes (5). Sólo para efectos de esta revisión se considerará como controlador a los ICS y no se hará referencia al uso de mon-

RESUMEN

El asma leve-moderada representa la mayor proporción de niños y adolescentes con asma en el mundo. Durante más de 25 años el tratamiento del asma permaneció invariable en torno al uso de controladores y rescatadores. Recientemente, diversas guías clínicas y sociedades científicas publicaron actualizaciones en torno al tratamiento de los pasos 1, 2 y 3 según GINA; existiendo diferencias y coincidencias entre ellas, a pesar de usar la misma evidencia publicada disponible. Este artículo de revisión se enfoca en tres quías clínicas qué por su popularidad y fácil acceso, son consultadas por diversos profesionales de la salud mostrando los principales cambios introducidos respecto a su edición previa y analizando brevemente la evidencia que existe detrás de estas recomendaciones. Finalmente se presenta un análisis de las principales limitaciones, contradicciones y aspectos aún por resolver.

Palabras claves: Asma, niños, adolescentes, tratamiento, guías clínicas.

ABSTRACT

Mild-moderate asthma represents the highest proportion of children and adolescents with asthma in the world. For more than 25 years, asthma treatment remained unchanged around the use of controllers and relievers. Recently, many clinical quidelines and scientific societies have published updates regarding the treatment of steps 1,2 and 3 according to GINA, with differences and coincidences between them, despite using the same available published evidence. This review article focuses on three clinical guides which, due to their popularity and easy access, are consulted by various health professionals, showing the main changes introduced compared to their previous edition and analyzing briefly the evidence behind these recommendations. Finally, an analysis of the main limitations, contradictions and aspects still to be resolved is presented. Keywords: Adolescent, traetment, clinical guidelines.

telukast ni a la terapia biológica. El objetivo de esta revisión es conocer los cambios más significativos publicados en las guías GINA 2021 (1), NHLBI 2020 (2) y GEMA 2021 (6) en torno al tratamiento del asma leve-moderada en niños y adolescentes.

\section{PASO 1}

Considera el tratamiento para el asma leve intermitente, en quienes los síntomas ocurren $<2$ días/mes sin factores de riesgo para desarrollar una exacerbación según GINA (1) (Tabla 1)

\section{Niños preescolares $\leq 5$ años}

Existe escasa evidencia nueva en este grupo de edad por la dificultad de establecer el diagnóstico de asma con certeza, por lo que no existen grandes cambios en relación al uso de controladores o rescatadores según necesidad 0 a libre demanda ( $p r n)$. GINA recomienda probar un tratamiento con ICS dosis bajas + SABA prn si presentan síntomas >2 veces/semana/ mes (1). El uso intermitente con ICS dosis alta + SABA prnestá recomendado por GINA para los ataques de sibilancias inducidas por virus (sin la presencia de síntomas entre episodios); de igual forma NHLBI sugiere la misma aproximación frente a la "sospecha de inicio" de una infección respiratoria viral (2). Existen 3 ensayos clínicos controlados (ECA) (7-9) a los que hace referencia esta recomendación (un total de 422 lactantes y preescolares con ICS durante 7-10 días); dos de ellos evaluaron además el posible impacto en el crecimiento, encontrando uno la pérdida de $5 \%$ en la velocidad de crecimiento lineal (8), mientras que el tercero no encontró ningún impacto. GEMA no introduce cambios en la versión 2021 para este grupo de edad (6).

\section{Niños escolares de 6-11 años (Figura 1)}

Existen dos opciones controladoras según GINA (1): a) el uso de ICS dosis bajas cuando se emplee SABA prn b) el uso de ICS dosis bajas diario (probablemente la menos convincente debido a la baja adherencia). Es-

Autor para correspondencia: Dr. Luis Enrique Vega-Briceño levega@udd.cl 
tas recomendaciones provienen del estudio TREXA (10) y de un ensayo abierto del "mundo real" con niños-adolescentes afroamericanos de 6-17 años (11). El primero empleó beclometasona (BDP) dosis bajas cuando se emplee SABA prn usando 2 inhaladores separados (843 pacientes con 5-18 años, durante 44 semanas), disminuyendo el riesgo de exacerbaciones severas en más de un tercio vs el grupo SABA prn. El segundo (206 pacientes con 6-17 años durante 52 semanas) demostró que el control del asma medido por el test del control del asma (ACT), la función pulmonar y la tasa de exacerbación, no fueron diferentes comparando BDP dosis bajas cuando use SABA prnvs el grupo BDP dosis baja diaria. Ambos estudios entregaron una menor dosis de ICS en el grupo ICS prn vs el grupo ICS dosis bajas diario, y por ello un menor impacto en el crecimiento (-1.1 cm de diferencia al final del estudio) (10). GEMA y NHLBI no introducen cambios en cuanto al uso de controladores o rescatadores en este grupo $(2,6)$.

\section{PASO 2}

Considera el tratamiento para niños y adolescente con asma leve persistente cuyos síntomas duran $\geq 2$ días/mes pero no en forma diaria (1).

\section{Niños preescolares $\leq \mathbf{5}$ años}

GINA recomienda como alternativa al uso regular de ICS dosis bajas diarias + SABA prn, el uso intermitente y breve de ICS dosis altas cuando se emplee SABA prnfrente al inicio de una infección respiratoria viral, consideran-

Tabla 1. Factores de riesgo para desarrollar una exacerbación por asma (incluso en pacientes con pocos síntomas) (1)

\begin{tabular}{|l|l|}
\hline Elevado consumo de SABA; no uso de ICS & Exposición a alergenos si está sensibilizado \\
\hline Mala técnica inhalatoria & Desórdenes psicológicos mayores \\
\hline Pobre adherencia & Problemas socioeconómicos \\
\hline Obesidad & VEF1 $60 \%$ predicho \\
\hline Rinosinusitis crónica & Elevada reversibilidad a SABA \\
\hline Reflujo gastroesofágico & Eosinófilos elevados en sangre \\
\hline Alergia alimentaria confirmada & FENO elevado (adultos usando ICS) \\
\hline Embarazo & Alguna vez en UCl o conectado a VM por asma \\
\hline Exposición a tabaco & $\geq 1$ exacerbación severa el año previo \\
\hline Contaminación ambiental & \\
\hline
\end{tabular}

SABA: $\beta 2$ agonista de acción corta; ICS: corticoide inhalado; VEF1: volumen espiratorio forzado en el primer segundo FENO: fracción exhalada de óxido nítrico; UCl: unidad de cuidados intensivos; VM: ventilación mecánica. Tomado y adaptado de: Global Initiative for Asthma (GINA). Global Strategy for Asthma Management and Prevention 2021. Disponible http://www.ginasthma.org/ acceso Junio 2021.

do además el uso de ICS dosis bajas diarias por 3 meses en aquellos con uso de SABA durante $\geq 3$ episodios de sibilancias/año (1). La evidencia proviene de tres ECA ya comentados (7-9) GEMA y NHLBI no introducen cambios en este grupo de edad $(2,6)$

\section{Niños escolares de 6-11 años (Figura 1)}

Existen dos nuevas recomendaciones en torno al uso de controladores para este grupo que provienen de dos estudios ya comentados $(10,11)$. La primera es el uso de ICS dosis bajas cuando se emplee SABA prn en inhaladores separados; sin embargo, GEMA y NHLBI no hacen esta recomendación $(2,6)$. La segunda nueva recomendación es el uso de terapia inmunológica, aspecto no abordado en esta revisión.

\section{Adolescentes $\geq 12$ años (Figura 2)}

Como ya se mencionó, GINA recomienda en el track 1 la combinación ICS/FT dosis bajas prn como la opción controladora y rescatadora preferida (1). La evidencia indirecta proviene de los estudios SYGMA $(15,16)$ ya comentados. Ambos estudios demostraron que el número

\section{TRATAMIENTO DEL ASMA LEVE-MODERADA EN NIÑOS 6-11 AÑOS SEGÚN GINA}

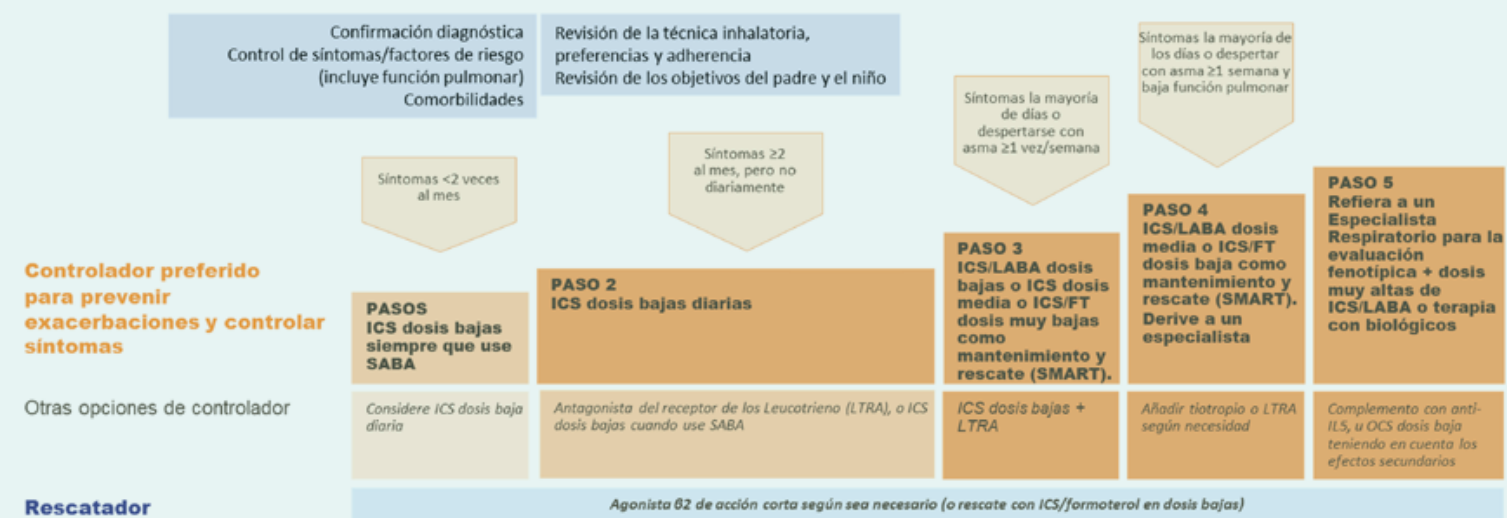

Figura 1. Tratamiento escalonado del asma en niños menores de 12 años según GINA 2021

SABA: $\beta 2$ agonista de acción corta; ICS: corticoide inhalado; FT: formoterol; LTRA: anti-leucotrieno; OCS: corticoides orales; SMART: tratamiento de mantenimiento y rescate. Tomado y adaptado de: Global Initiative for Asthma (GINA). Global Strategy for Asthma Management and Prevention 2021. Disponible http:// www.ginasthma.org/ acceso Junio 2021. 
de exacerbaciones anuales y la tasa de exacerbaciones graves no fueron inferiores entre el grupo ICS/FT prn vs ICS dosis baja diaria. Los beneficios del uso regular de ICS (reducción de exacerbaciones severas, hospitalización y mortalidad) son bien reconocidos $(12,17,18)$; pero el cumplimiento y adherencia al tratamiento diario regular es muy importante, aspecto que no ocurre en los adolescentes especialmente con asma leve-moderada (19). En el track 2 se recomienda el esquema tradicional del uso de ICS dosis bajas diario. El novedoso régimen del track 1 no es recomendado por CEMA ni NHLBI: ambos recomiendan el uso regular de ICS dosis bajas diario + SABA prno ICS dosis bajas cuando se emplee SABA prn $(2,6)$. La evidencia proviene de dos estudios anteriormente comentados $(10,11)$. Esta opción de tratamiento intermitente tiene los mismos efectos sobre el control del asma y la frecuencia de exacerbaciones vs el grupo con ICS dosis bajas diaria presentando efectos secundarios infrecuentes; sin embargo, recordemos que este grupo de pacientes tienen una baja 0 alta percepción de sus síntomas pudiendo no ser adecuados candidatos para un tratamiento intermitente flexible con ICS (19). Por su parte GEMA y NHLBI no introducen estos cambios $(2,6)$. Esta revisión no evalúa el rol de la inmunoterapia, aspecto si recomendado en el paso 2 de GINA 2021 (1).

\section{PASO 3}

Considera el tratamiento para niños y adolescentes con asma persistente moderada, definida por la presencia de síntomas diarios frecuentes o por la presencia de despertares nocturnos 2 1v/semana según GINA (1).

\section{Niños preescolares $\leq 5$ años}

De manera interesante GINA y GEMA no establecen nuevas recomendaciones para este grupo (1,6); sin embargo, NHLBI agrega una novedosa y a la vez extraña recomendación en relación al tratamiento controlador (2). Para niños $\leq 3$ años, sugiere usar ICS/LABA dosis bajas diarias como controlador + SABA prn. No existen estudios que evalúen la combinación ICS/ FT en <4 años; sin embargo, existen 3 ECA que si utilizaron la combinación fluticasona propionato $(F P)+$ salmeterol (SAL) en un sólo inhalador diario + SABA prn en niños <5 años (20-22). El primero demostró en 6208 niños entre 4-11 años que la combinación FP/SAL durante 26 semanas fue no inferior en términos de eficacia para prevenir las exacerbaciones graves que requirieron hospitalización vs la misma dosis de FP sola (hazard ratio: 1,28 IC: 0,73-2,27), concluyendo que la combinación FP/SAL tenía el mismo perfil de seguridad que el ICS solo (20). El segundo estudio incluyó 300 niños de 8 meses a 4 años empleando FP/SAL 80 mcg/25 mcg vs la misma dosis de FP por 8 semanas en donde, la combinación de FP/SAL no demostró mayor eficacia en el score de síntomas vs FP sola (cambio promedio -3.97 para FP/SAL vs -3.01 para FP), teniendo ambos esquemas el mismo perfil de seguridad y siendo el primer ECA que evaluó esta combinación en lactantes (21). El último estudio, observacional, utilizó una combinación de FP/SAL en 796 niños < 5 años durante 12 meses en promedio, mostrando una reducción del $71 \%$ de los síntomas relacionados al ejercicio, $81 \%$ en síntomas nocturnos y $89 \%$ en la tasa de hospitalización; siendo bien tolerada y más efectiva que otros controladores (22). Debe recordarse que esta indicación mencionada no está aprobada según el fabricante (23).

\section{Niños de 6-11 años (Figura 1)}

GINA y NHLBI publicaron una nueva recomendación en torno al uso de ICS/FT en un solo inhalador como controlador y rescatador como la opción preferida en este grupo $(1,2)$. Hay 4 ECA (24-27) para esta recomendación, dos de los cuales incluyeron los mismos niños menores de 11 años $(24,27)$. Todos los estudios encontraron una disminución significativa en el número de exacerbaciones graves (>70\%), control de síntomas diurnos y nocturnos e incremento de la función pulmonar; prolongando el tiempo de la primera exacerbación que requirió atención médica y empleando menores dosis de ICS; dos estudios mostraron un mayor crecimiento en el grupo ICS/FT (+1 cm/año IC: 0.3 1.7; $p=0.0054$ ) empleando dosis bajas como controlador y rescate vs ICS dosis altas diario + SABA prn $(24,27)$. GEMA no introduce estos cambios ni recomendaciones relacionadas (6).

\section{Adolescentes $\geq 12$ años (Figura 2)}

En el track 1 GINA recomienda la combinación ICS/FT dosis bajas diarias y prnsiendo la opción controladora y rescatadora preferida (1). Esta preferencia de controlador y rescatador es similar en NHLBI (2). Sin embargo, mencionan que la combinación fija de ICS/FT no debe usarse como rescate en aquellos adolescentes que usan otras combinaciones ICS/LABA + SABA prn (track 2) (1). Existe evidencia que demuestra

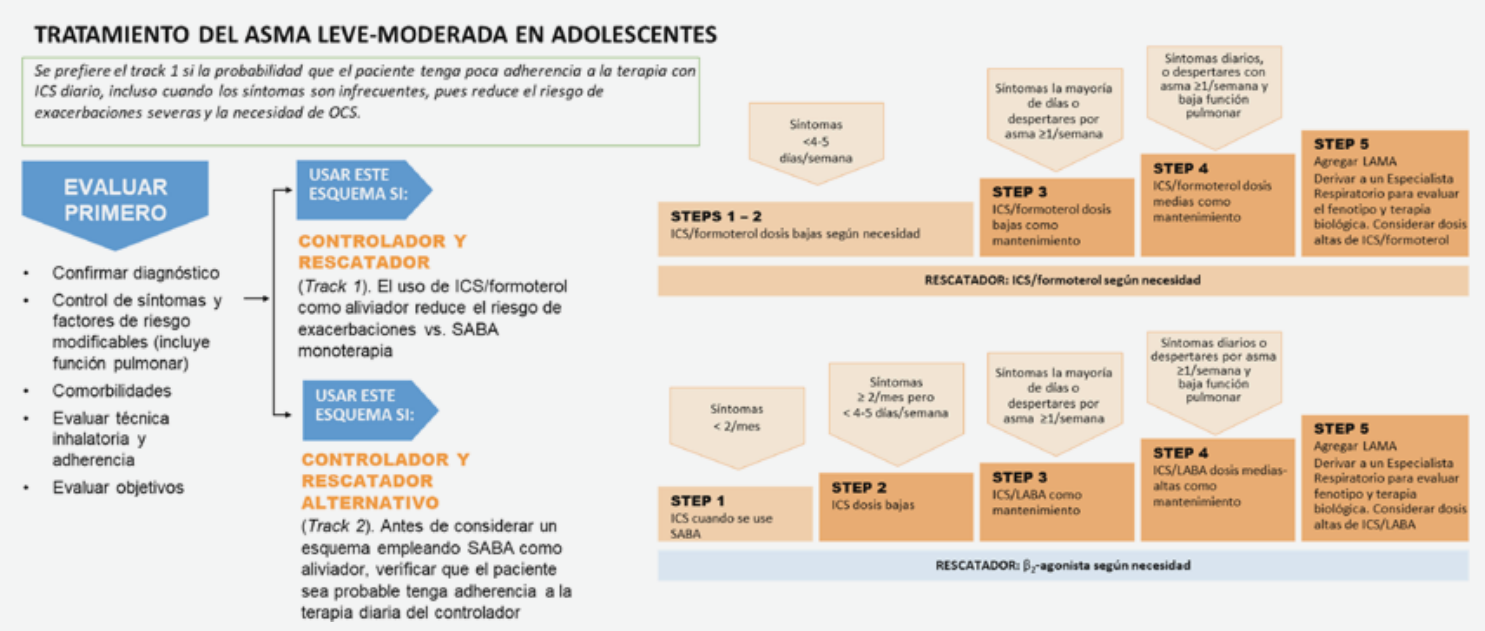

Figura 2. Tratamiento escalonado del asma en niños mayores de 12 años según CINA 2021. SABA: $\beta 2$ agonista de acción corta; ICS: corticoide inhalado; FT: formoterol; OCS: corticoides orales. Tomado y adaptado de: Global Initiative for Asthma (CINA). LAMA: Agonista muscarínico de acción prolongada. Global Strategy for Asthma Management and Prevention 2021. Disponible http://www.ginasthma.org/ acceso Junio 2021. 
que ICS/FT usado como controlador y rescatador en 1847 adolescentes provenientes de 6 ECA reduce significativamente las exacerbaciones severas y proporciona un nivel de control similar al grupo ICS/FT dosis bajas diario o el grupo ICS dosis altas + SABA prn (28). La opción ICS/FT como controlador y rescatador podría no ser necesaria para los pacientes con asma bien controlada con ICS/LABA dosis baja diaria + SABA prn (1). Por otro lado, sugieren que los pacientes con una exacerbación severa el año previo podrían ser buenos candidatos para el uso ICS/FT como controlador y rescatador (24). Tanto BDP/FT (29) o BUD/FT se recomiendan como tratamiento controlador y rescatador en un solo inhalador (reducción del riesgo de la primera exacerbación de 36\%); aunque los estudios en niños y adolescentes fueron hechos con BUD/FT (24-28). La dosis máxima empleada en forma diaria de FT no excedió los 54 $\mu \mathrm{g}$ en $>12$ años (25-27). Esta revisión no evalúa el rol de la inmunoterapia incorporada en este grupo de pacientes con asma moderada.

\section{LIMITACIONES Y CONTRADICCIONES}

Primero, las recomendaciones publicadas son exactamente eso: "recomendaciones" de un grupo de expertos que interpretan la misma evidencia publicada sin una metodología estandarizada como la denominada GRADE (sólo GEMA empleó este método de forma incompleta); por ello, existen diferencias sustan- ciales entre las guías (Tabla 2). Para efectos de esta revisión sólo se consideraron 3 guías clínicas (GINA, GEMA, NHLBI). Segundo, todas las intervenciones con la combinación ICS cuando se emplee SABA prn o ICS/FT prn provienen de ECA con una duración no superior a las 52 semanas; es decir, no se evaluó el impacto de estas nuevas intervenciones pasado el periodo de estudio, lo que significa que se desconoce la evolución clínica o el desenlace de cada participante una vez terminado el estudio. Mas aún, todas las intervenciones emplearon el mismo end point primario o desenlace: tasa de exacerbación (que sin duda es importante) o control del asma; sin haber evaluado todos en algún momento: a) la remisión o control de la inflamación, b) el impacto o incremento de la función pulmonar, c) el impacto o control de la hiperreactividad bronquial, d) el impacto sobre el puntaje ACT u otro score clínico similar, e) la seguridad en términos de sobrevida, y f) el impacto de costo-efectividad. Todos los aspectos señalados anteriormente, han sido logrados con el uso regular de ICS o ICS/LABA. Tercero, el uso de algún $\beta$-agonista (SABA o LABA) en forma diaria o intermitente, caracteriza al paciente con asma mal controlada y resulta razonable inferir que existe una inflamación no resuelta; por ello, surge la pregunta ¿por qué un paciente con asma leve-moderada en un ECA requiere dosis extra de ICS/FT para evitar una exacerbación grave?; ¿existirá realmente control de su condición de base denomina- da inflamación?; como bien se menciona en muchos estudios, ello está bajo el paragua de la percepción de cada paciente quien decidirá administrar un número de inhalaciones extras que considera necesario. Cuarto, se desconoce el perfil de seguridad de la combinación ICS/FT prn a largo plazo; por ello parece apropiado seguir invirtiendo en educación y adherencia para no transferir la responsabilidad al paciente de decidir cuando el considera usar un ICS o ICS/ LABA prn con resultados muy alentadores a corto plazo, pero olvidando por un instante que el asma es una condición inflamatoria crónica. Finalmente, quinto, parece contradictorio reconocer por un lado que los adolescentes con asma leve tienen la peor tasa de adherencia al uso regular de controladores, pero a la vez empoderarlos a decidir cuándo deben emplearlos; olvidando nuevamente que representan el grupo con la peor percepción de síntomas, enfermedad y gravedad, subrayando el concepto que el asma es una condición invisible (30).

\section{CONCLUSIONES}

Tanto las guías de asma de GINA, GEMA y NHLBI publicaron recomendaciones en función de la evidencia reciente disponible. Existen dos nuevas propuestas de tratamiento controlador y rescatador para el asma leve-moderada en niños y adolescentes: a) ICS/FT en un sólo inhalador, b) ICS dosis bajas cuando se emplee SABA prn; sin embargo, estas recomendacio-

Tabla 2. Cambios en el tratamiento del asma publicados según tres guías internacionales.

\begin{tabular}{|c|c|c|c|c|}
\hline & & GINA 2021 (1) & GEMA 2021 (6) & NHLBI 2020 (2) \\
\hline \multirow{3}{*}{$\begin{array}{c}\text { Paso } \\
1\end{array}$} & $>12$ años & $\begin{array}{l}\text { ICS/FT dosis bajas como rescate(") } \\
\text { ICS dosis bajas + SABA como rescate }\end{array}$ & ICS/FT dosis bajas como rescate & No hay cambios \\
\hline & 6-11años & ICS dosis bajas + SABA como rescate & No hay cambios & No hay cambios \\
\hline & $\leq 5$ años & No hay cambios & No hay cambios & $\begin{array}{l}\text { SABA como rescate + ICS diario al } \\
\text { inicio de una infección respiratoria viral }\end{array}$ \\
\hline \multirow{3}{*}{$\begin{array}{c}\text { Paso } \\
2\end{array}$} & $>12$ años & ICS/FT dosis bajas como rescate & ICS/FT dosis bajas como rescate & ICS dosis bajas + SABA como rescate \\
\hline & 6-11años & ICS dosis bajas + SABA como rescate & No hay cambios & No hay cambios (**) \\
\hline & $\leq 5$ años & No hay cambios & No hay cambios & No hay cambios \\
\hline \multirow{3}{*}{$\begin{array}{c}\text { Paso } \\
3\end{array}$} & $>12$ años & $\begin{array}{l}\text { ICS/FT dosis bajas mantención y } \\
\text { rescate }\end{array}$ & ICS/FT dosis bajas como rescate & $\begin{array}{l}\text { ICS/FT dosis bajas mantención y } \\
\text { rescate }\end{array}$ \\
\hline & 6-11 años & $\begin{array}{l}\text { ICS/FT dosis bajas mantención y } \\
\text { rescate }\end{array}$ & No hay cambios & $\begin{array}{l}\text { ICS/FT dosis bajas mantención y } \\
\text { rescate }\end{array}$ \\
\hline & $\leq 5$ años & No hay cambios & No hay cambios & $\begin{array}{c}\text { ICS/LABA dosis bajas + SABA rescate } \\
\text { (<3 años) } \\
\text { ICS/FT dosis bajas mantención y } \\
\text { rescate }(>4 \text { años })\end{array}$ \\
\hline
\end{tabular}

Leyenda: ICS: corticoide inhalado; FT: formoterol; SABA: beta agonista de acción corta; prn: a libre demanda; (*): GINA fusiona los pasos 1 y 2 en uno solo en >12 años; $\left({ }^{*}\right)$ : sugieren agregar inmunoterapia la cual no es abordado en esta revisión. 
nes no condicen con los pasos del tratamiento según cada grupo de edad, lo que traduce una discrepancia entre los autores que sugieren ambos esquemas. Si bien las guías clínicas deberían facilitar la elección de la mejor opción controladora y rescatadora del asma leve-moderada en niños y adolescentes, parece que la interpretación de la misma evidencia puede diferir dependiendo de quienes sean sus autores; produciendo más confusión que claridad a la hora de prescribir uno u otro esquema. A la luz de esta revisión parece necesario una estrategia de tratamiento personalizada no sólo considerando la severidad del asma, sino también la importancia de la educación y adherencia en todos los pasos, la percepción de síntomas de cada paciente y el impacto futuro de la enfermedad y los costos asociados a estos esquemas de tratamientos intermitentes

Conflicto de interés: El autor declara ser Asesor Medico GSK.

\section{REFERENCIAS}

1. Global Initiative for Asthma (GINA). Global strategy for asth ma management and prevention (Updated 2021). Available from: www.ginasthma.org. Acceso junio, 2021.

2. A report from the National Asthma Education and Prevention Program Coordinating Committee Expert Panel Working Group. 2020 Focused Updates to The Asthma Management Guidelines. National Heart, Lung, and Blood Institute, National Institutes of Health; 2020. Available from: www.nhlbi.nih.gov. acceso Junio, 2021

3. Fitzpatrick AM, Bacharier LB, Jackson DJ, Szefler S, Beigelman A, Cabana M, Covar R, et al. Heterogeneity of mild to moderate persistent asthma in children: confirmation by latent class analysis and association with 1-year outcomes. J Allergy Clin Immunol Pract 2020;8(8):2617-27. DOI: 10.1016/j.jaip.2020.02.032

4. Papadopoulos NG, Čustović A, Cabana MD, Dell SD, Deschildre A, Hedlin G, et al. Pediatric asthma: an unmet need for more effective, focused treatments. Pediatr Allergy Immunol 2019;30(1):7-16. DOI: 10.1111/pai.12990

5. FDA. FDA Requires Boxed Warning About Serious Menta Health Side Effects for Asthma and Allergy Drug Montelukast (Singulair); Advises Restricting Use for Allergic Rhinitis. FDA; 2020. Acceso Mayo 2021

6. Gema 5.1. Guía española para el manejo del asma [Internet] España: Sociedad Española de Neumología y Cirugía Torácica (SEPAR); c2020 [citada junio 2021]. Disponible en https://neumoped.org/actualizacion-gema-5-1/

7. Bacharier LB, Phillips BR, Zeiger RS, Stanley J, Martinez F, Lemanske $R$, et al. Episodic use of an inhaled corticosteroid or leukotriene receptor antagonist in preschool children with moderate-to-severe intermittent wheezing. J Allergy Clin Immunol 2008;122(6):1127-35.e8. DOI: 10.1016/j. jaci.2008.09.029

8. Ducharme FM, Lemire C, Noya FJ, Davis GM, Alos N, Leblond $\mathrm{H}$, et al. Preemptive use of high-dose fluticasone for virus-induced wheezing in young children. N Engl I Med 2009;360(4):339-53. DOI: 10.1056/NEJMoa0808907

9. Svedmyr J, Nyberg E, Thunqvist P, Asbrink-Nilsson E, Hedlin G. Prophylactic intermittent treatment with inhaled corticosteroids of asthma exacerbations due to airway infections in toddlers. Acta Paediatr 1999;88(1):42-7. DOI: 10.1080/08035259950170583

10. Martinez FD, Chinchilli VM, Morgan WJ, Boehmer SJ, Lemanske RF, Mauger D, et al. Use of beclomethasone dipropionate as rescue treatment for children with mild persistent asthma (TREXA): a randomised, double-blind, placebo-controlled trial. Lancet 2011;377(9766):650-7. doi: 10.1016/S0140-6736(10)62145-9

11. Sumino K, Bacharier LB, Taylor J, Chadwick-Mansker K, Curtis V, Nash A, et al. A pragmatic trial of symptom-based inhaled corticosteroid used in African-American Children with Mild Asthma. J Allergy Clin Immunol Pract 2020;8(1):17685.e2. DOI: 10.1016/j.jaip.2019.06.030

12. Suissa S, Ernst P, Benayoun S, Baltzan M, Cai B. Low-dose inhaled corticosteroids and the prevention of death from asthma. N Engl J Med 2000;343(5):332-6. DOI: 10.1056/ NEJM200008033430504

13. Suissa S, Ernst P, Kezouh A. Regular use of inhaled corticosteroids and the long-term prevention of hospitalisation for asthma. Thorax 2002;57(10):880-4. DOI: 10.1136/thorax.57.10.880

14. Reddel HK, Ampon RD, Sawyer SM, Peters MJ. Risks associated with managing asthma without a preventer: urgent healthcare, poor asthma control and over-the-counter reliever use in a cross-sectional population survey. BMJ Open 2017;7(9):e016688. DOI: 10.1136/bmjopen-2017-016688

15. O'Byrne PM, FitzGerald JM, Bateman ED, Barnes PJ, Zhong N, Keen C, et al. Inhaled Combined Budesonide-Formoterol as Needed in Mild Asthma. N Engl J Med
2018:378(20):1865-76 doi:10.1056/NEJMoa1715274

16. Bateman ED, Reddel HK, O'Byrne PM, Barnes PJ, Zhong N, Keen C, et al. As-Needed Budesonide-Formoterol versus Maintenance Budesonide in Mild Asthma. N Engl J Med 2018;378(20):1877-87.DOI: 10.1056/NEJMoa1715275

17. Pauwels RA, Pedersen S, Busse WW, Tan WC, Chen Y, Ohlsson $\mathrm{S}$, et al. Early intervention with budesonide in mild persistent asthma: a randomised, double-blind trial. Lancet 2003;361(9363):1071-76. DOI: 10.1016/S01406736(03)12891-7

18. Kaiser SV, Huynh T, Bacharier LB, Rosenthal JL, Bakel LA, Parkin $\mathrm{P}$, et al. Preventing exacerbations in preschoolers with recurrent wheeze: a meta-analysis. Pediatrics [Internet] 2016;137(6):e20154496. DOI: 10.1542/peds.20154496

19. Bitsko MJ, Everhart RS, Rubin BK. The adolescent with asthma. Paediatr Respir Rev 2014;15(2):14-53. DOI: 10.1016/j. prrv.2013.07.003

20. Stempel DA, Szefler SJ, Pedersen S, Zeiger RS, Yeakey AM, Lee L, et al. Safety of Adding Salmeterol to Fluticasone Propionate in Children with Asthma. N Engl J Med 2016;375(9):840-9. DOI: 10.1056/NEJMoa1606356

21. Yoshihara S, Tsubaki T, Ikeda M, Lenney W, Tomiak R, Hattori $\mathrm{T}$, et al. The efficacy and safety of fluticasone/salmeterol compared to fluticasone in children younger than four years of age. Pediatr Allergy Immunol 2019;30(2):195-203. DOI: 10.1111/pai.13010

22. Hatziagorou E, Kouroukli $E$, Galogavrou M, Papanikolaou D, Terzi D, Anagnostopoulou P, et al. Efficacy and safety of the combination fluticasone propionate plus salmeterol in asthmatic preschoolers: an observational study. J Asthma 2019;56(6):573-80. DOl: 10.1080/02770903.2018.1474923

23. https://gskpro.com/content/dam/global/hcpportal/es_ AR/pdfs/homepage/products/seretide/ar-portal-IP-seretideaerosol2017.pdf acceso junio 2021

24. O'Byrne PM, Bisgaard H, Godard PP, Pistolesi M, Palmqvist $M$, Zhu Y, et al. Budesonide/formoterol combination therapy as both maintenance and reliever medication in asthma. Am J Respir Crit Care Med 2005;171(2):129-36. DOI: 10.1164/ rccm.200407-8840C

25. Rabe KF, Pizzichini E, Stallberg B, Romero S, Balanzat AM, Atienza $T$, et al. Budesonide/formoterol in a single inhaler for maintenance and relief in mild-to-moderate asthma: a randomized, double-blind trial. Chest 2006;129(2):246-56. DOI: 10.1378/chest.129.2.246

26. Scicchitano R, Aalbers R, Ukena D, Manjra A, Fouquert L, Centanni S, et al. Efficacy and safety of budesonide/formoterol single inhaler therapy versus a higher dose of budesonide in moderate to severe asthma. Curr Med Res Opin 2004;20(9):1403-18. DOI: 10.1185/030079904X2051

27. Bisgaard H, Le Roux P, Bjamer D, Dymek A, Vermeulen JH, Hultquist C. Budesonide/formoterol maintenance plus reliever therapy: a new strategy in pediatric asthma. Chest 2006;130(6):1733-43. DOI: 10.1378/chest.130.6.1733

28. Jorup C, Lythgoe D, Bisgaard H. Budesonide/formoterol maintenance and reliever therapy in adolescent patients with asthma. Eur Respir J 2018;51(1):1701688. doi: 10.1183/13993003.01688-2017

29. Papi A, Corradi M, Pigeon-Francisco C, Baronio R, Siergiejko Z, Petruzzelli S, et al. Beclometasone-formoterol as maintenance and reliever treatment in patients with asthma: a double-blind, randomised controlled trial. Lancet Respir Med 2013;1(1):23-31. DOI: 10.1016/S2213-2600(13)70012-2

30. Vega-Briceño L. Asma leve: un problema de salud pública invisible. Neumol Pediatr 2021;16(2):90-91. D0I: 10.51451/ np.v16i2.330 